\title{
Research Article \\ On the Nonlinear Theory of Micromorphic Thermoelastic Solids
}

\author{
C. Galeş \\ Faculty of Mathematics, University of Iaşi, 700506 Iaşi, Romania \\ Correspondence should be addressed to C. Galeş, cgales@uaic.ro
}

Received 27 September 2010; Accepted 18 November 2010

Academic Editor: J. Rodellar

Copyright (C) 2010 C. Galeş. This is an open access article distributed under the Creative Commons Attribution License, which permits unrestricted use, distribution, and reproduction in any medium, provided the original work is properly cited.

This work aims to study some dynamical problems in the framework of nonlinear theory of micromorphic thermoelastic solids. First, the continuous dependence of smooth admissible thermodynamic processes upon initial state and supply terms is investigated in the region of state space where the internal energy is a convex function and the elastic material behaves as a definite conductor of heat. Then, a uniqueness result is demonstrated.

\section{Introduction}

Motivated by experimental studies, various continuous models of deformable bodies have been proposed in literature in order to describe the thermomechanical behavior of media with microstructure. In the micromorphic theory introduced by Eringen and Şuhubi [1] and Eringen [2], a material body is envisioned as a collection of a large number of deformable particles (subcontinua or microcontinua). Each particle possesses finite size and directions representing its microstructure. The microdeformation gives rise to extra degrees of freedom. Thus, the particle has nine independent degrees of freedom describing both rotations and stretches, in addition to the three classical translational degrees of freedom of its center.

Many deformable solids point to the necessity for the incorporation of micromotions into mechanics. Porous solids with deformable grains and pores, composites, polymers with deformable molecules, crystals, solids with microcracks, dislocation and disclinations, and biological tissues (bones and muscles) are just a few examples of deformable solids which require the degrees of freedom given by the micromorphic theory. As a consequence, the micromorphic mechanics is the subject of detailed studies both from theoretical and practical reasons. In the linear context, uniqueness theorems have been proved by Soós [3] and Ieşan [4], variational principles have been established by Maugin [5] and Nappa [6], applications 
to earthquake problems have been suggested by Teisseyre [7], Dresen et al. [8], and Teisseyre et al. [9], plane harmonic waves have been studied by Eringen [2], reciprocal and existence theorems have been demonstrated by Ieşan [4], and material constants for isotropic materials have been determined by Chen and Lee [10].

On the other hand, the theory has been generalized to mixtures of micromorphic materials by Twiss and Eringen [11, 12], to higher-grade materials by Eringen [13], and to electromagnetic micromorphic thermoelastic solids by Eringen [14]. Moreover, the constitutive theory of micromorphic thermoplasticity has been formulated by Lee and Chen [15], the concept of material forces was extended to micromorphic theory by Lee and Chen [16], the problem of heat flow in a micromorphic continua with microtemperatures has been investigated by Ieşan and Nappa [17].

This paper deals with the nonlinear micromorphic thermoelasticity. The main purpose is to investigate the stability of smooth thermodynamic processes. In this sense, we use the method developed for nonlinear thermoelastic solids which are nonconductor of heat by Dafermos [18] and updated later by Chiriţă [19] to heat-conducting thermoelastic materials. In the general context of heat-conducting nonlinear micromorphic solids, we prove the continuous dependence of smooth admissible thermodynamic processes upon initial state and supply terms. We present also a uniqueness theorem. Both results are obtained in the region of state space where the internal energy is a convex function and the elastic material behaves as a definite conductor of heat.

We recall that the Dafermos method has been utilized recently to prove continuous dependence results for nonconductor-of-heat mixtures [20-22] and for materials with voids [23].

The paper is organized as follows. In the next section, we recall the basic equations of the nonlinear theory of micromorphic thermoelasticity. Then, in Section 3, we use the consequences of the second law of thermodynamics to prove a uniqueness theorem and the continuous dependence of smooth thermodynamic processes upon initial state and body loads.

\section{Basic Formulation}

We consider a micromorphic continuum, and we assume that at time $t_{0}$ the body occupies the region $B$ of the Euclidean three-dimensional space and is bounded by a piecewise smooth surface $\partial B$. Following [2], a point $C$ in the body is characterized by its rectangular coordinates $X_{1}, X_{2}, X_{3}$, in a fixed system of rectangular Cartesian axes $O X_{K}, K=1,2,3$, and a deformable vector $\boldsymbol{\Xi}$. One writes $C(\mathbf{X}, \boldsymbol{\Xi})$. Deformation carries $C(\mathbf{X}, \boldsymbol{\Xi})$ to $c(\mathbf{x}, \boldsymbol{\xi})$ in the spatial configuration at time $t$, where $x_{1}, x_{2}$, and $x_{3}$ are the rectangular coordinates with respect to a new coordinate Cartesian system $o x_{i}, i=1,2,3$, and $\xi$ is the vector attached to $c$. The motion can be expressed as

$$
x_{i}=x_{i}(\mathbf{X}, t), \quad \xi_{i}=\chi_{i K}(\mathbf{X}, t) \Xi_{K}, \quad \mathbf{X} \in B, t \in I,
$$

where, and henceforth, the summation convention on repeated indices is understood and $I=\left[t_{0}, t_{1}\right), t_{1}>t_{0}$. 
The inverse motion can be written as

$$
X_{K}=X_{K}(\mathbf{x}, t), \quad \Xi_{K}=\mathfrak{X}_{K i}(\mathbf{x}, t) \xi_{i},
$$

where

$$
\chi_{i K} \mathfrak{X}_{K j}=\delta_{i j}, \quad \chi_{i K} \mathfrak{X}_{L i}=\delta_{K L}
$$

In what follows $\dot{f}$ denotes the material time rate of $f$. Moreover, we use the notations $f_{K}=\partial f / \partial X_{K}$ and $f_{i}=\partial f / \partial x_{i}$.

The balance laws of micromorphic continua can be expressed as follows [1, 2]:

(i) the conservation of mass:

$$
\rho_{0}=J \rho \text { or } \dot{\rho}+\rho v_{i, i}=0,
$$

where $\rho_{0}(\mathbf{X})$ and $\rho(\mathbf{x}, t)$ are the mass densities at time $t_{0}$ and at present time, respectively, $J=\operatorname{det}\left(\partial x_{i} / \partial X_{K}\right)$, and $v_{i}$ are the components of velocity vector;

(ii) the conservation of micromorphic inertia:

$$
i_{k l}=I_{K L} X_{k K} \chi_{l L}, \quad I_{K L}=i_{k l} \mathfrak{X}_{K k} \mathfrak{X}_{L l} \quad \text { or } \quad \frac{d i_{k l}}{d t}=i_{k m} v_{l m}+i_{l m} v_{k m},
$$

where $v_{k l}$ is the microgyration tensor defined by

$$
v_{i j}=\dot{X}_{i K} \mathfrak{X}_{K j},
$$

and $I_{K L}(\mathbf{X})$ are the components of the inertia stress tensor at time $t_{0}$ and $i_{k l}(\mathbf{x}, t)$ are the components of the microinertia tensor at time $t$. Clearly, $I_{K L}(\mathbf{X})$ and $i_{k l}(\mathbf{x}, t)$ are symmetric and positive definite;

(iii) the balance of momentum:

$$
t_{j i, j}+\rho f_{i}=\rho \dot{v}_{i}
$$

(iv) the balance of moment of momentum:

$$
m_{k i j, k}+t_{j i}-s_{j i}+\rho l_{i j}=\rho \sigma_{i j},
$$


(v) the balance of energy:

$$
\rho \dot{e}=t_{j i} v_{i, j}+m_{k i j} v_{i j, k}+\left(s_{j i}-t_{j i}\right) v_{i j}+q_{i, i}+\rho r,
$$

where $\mathbf{t}, \mathbf{m}, \mathbf{s}, \boldsymbol{\sigma}, e, \mathbf{q}$ are the stress tensor, moment stress tensor, microstress average, spin inertia, internal energy, and heat input, respectively, and $\mathbf{f}, \mathbf{l}, r$ are the body force, external body moment, and heat source, respectively. The tensor $\mathbf{s}$ is symmetric, that is, $s_{i j}=s_{j i}$. The spin inertia $\sigma_{i j}$ may be expressed as

$$
\sigma_{i j}=I_{K L} \ddot{X}_{i K} X_{j L}=i_{m j}\left(\dot{v}_{i m}+v_{i n} v_{n m}\right) .
$$

The second law of thermodynamics is written as

$$
\rho \theta \dot{\eta}-q_{i, i}-\rho r+\frac{1}{\theta} q_{i} \theta_{, i} \geq 0,
$$

where $\eta$ is the entropy density and $\theta$ is the absolute temperature.

The above formulation is described in detail in the book by Eringen [2]. Since we deal with micromorphic solids, we reformulate the basic equations in Lagrangean description. Thus, introducing the Piola-Kirchhoff tensors

$$
\begin{gathered}
T_{K i}=J X_{K, j} t_{j i}, \quad M_{K i L}=J X_{K, j} \mathfrak{X}_{L k} m_{j i k}, \quad Q_{K}=J X_{K, i} q_{i}, \\
S_{K i}=J X_{K, j} S_{j i}, \quad L_{i K}=\mathfrak{X}_{K j} l_{i j},
\end{gathered}
$$

and making the notation

$$
G_{i L}=\mathfrak{X}_{L j}\left[x_{j, K}\left(S_{K i}-T_{K i}\right)-x_{j M, K} M_{K i M}\right],
$$

then, with the help of the relations (2.4), (2.6), and (2.10) and the identity

$$
\left(\frac{x_{i, K}}{J}\right)_{, i}=0
$$

we obtain the equations of motion

$$
\begin{gathered}
T_{K i, K}+\rho_{0} f_{i}=\rho_{0} \ddot{x}_{i}, \\
M_{K i L, K}-G_{i L}+\rho_{0} L_{i L}=\rho_{0} I_{K L} \ddot{X}_{i K},
\end{gathered}
$$

the energy equation

$$
\rho_{0} \dot{e}=T_{K i} v_{i, K}+M_{K i L} \dot{X}_{i L, K}+G_{i K} \dot{X}_{i K}+Q_{K, K}+\rho_{0} r,
$$


and the inequality

$$
\rho_{0} \theta \dot{\eta}-Q_{K, K}-\rho_{0} r+\frac{1}{\theta} Q_{K} \theta_{, K} \geq 0
$$

Introducing Helmholtz's free energy $\psi$ as

$$
\psi=e-\theta \eta
$$

then, with the help of (2.15), the energy equation can be expressed as

$$
\frac{d}{d t}\left[\rho_{0}\left(\psi+\theta \eta+\frac{1}{2} v_{i} v_{i}+\frac{1}{2} I_{K L} \dot{X}_{i K} \dot{X}_{i L}\right)\right]=\left(T_{K i} v_{i}+M_{K i L} \dot{X}_{i L}+Q_{K}\right)_{, K}+\rho_{0}\left(f_{i} v_{i}+L_{i L} \dot{X}_{i L}+r\right) .
$$

Moreover, from (2.16), (2.17), and (2.18), we deduce

$$
-\rho_{0}(\dot{\psi}+\eta \dot{\theta})+T_{K \mathrm{i}} v_{i, K}+M_{K i L} \dot{X}_{i L, K}+G_{i K} \dot{X}_{i K}+\frac{1}{\theta} Q_{K} \theta_{, K} \geq 0 .
$$

The response of a micromorphic thermoelastic solid is characterized by the following constitutive equations:

$$
\begin{gathered}
\psi=\widehat{\psi}(\mathfrak{L}), \quad T_{L j}=\widehat{T}_{L j}(\mathfrak{L}), \quad G_{j L}=\widehat{G}_{j L}(\mathfrak{L}), \quad M_{L j P}=\widehat{M}_{L j P}(\mathfrak{L}), \\
\eta=\widehat{\eta}(\mathfrak{L}), \quad Q_{L}=\widehat{Q}_{L}(\mathfrak{L}),
\end{gathered}
$$

where

$$
\mathfrak{L}=\left\{x_{i, K,} X_{i K}, X_{i K, M}, \theta, \theta_{, K} ; X_{K}\right\}
$$

and $\widehat{\psi}, \widehat{T}_{L j}, \widehat{G}_{j L}, \widehat{M}_{L j P}, \widehat{\eta}$, and $\widehat{Q}_{L}$ are sufficient smooth functions.

We assume that there is no kinematical constraint. Then, it follows from the inequality (2.20) that

$$
\begin{gathered}
\psi=\widehat{\psi}\left(x_{i, K}, x_{i K}, x_{i K, M}, \theta ; X_{K}\right), \quad Q_{L}=\widehat{Q}_{L}(\mathfrak{L}), \\
T_{L j}=\rho_{0} \frac{\partial \widehat{\psi}}{\partial x_{j, L}}, \quad G_{j L}=\rho_{0} \frac{\partial \widehat{\psi}}{\partial x_{j L}}, \quad M_{K j L}=\rho_{0} \frac{\partial \widehat{\psi}}{\partial \chi_{j L, K}}, \quad \eta=-\frac{\partial \widehat{\psi}}{\partial \theta}, \\
\widehat{Q}_{L}\left(x_{i, K}, x_{i K}, x_{i K, M}, \theta, 0 ; X_{K}\right)=0 .
\end{gathered}
$$

The inequality (2.20) reduces to

$$
\widehat{Q}_{K} \theta_{, K} \geq 0
$$




\section{Uniqueness and Continuous Dependence}

In this section, we establish a uniqueness result and the continuous dependence of smooth thermodynamic processes upon initial state and supply terms.

We assume that $B$ is a bounded region and that $\partial B$ is sufficiently regular to assure the common laws of transformation of surface integrals. We will employ the following notations: $N_{K}$ are the components of the unit outward normal vector to the surface $\partial B$; $I$ denotes the time interval $\left[t_{0}, t_{1}\right)$, where $t_{1}$ may be infinity; the symbol $|\cdot|$ denotes a norm, either in Euclidean vector space or in a tensor space, while $\|\cdot\|_{L^{2}(B)}$ denotes the $L^{2}$-norm.

Definition 3.1. By a thermodynamic process on $B \times I$ one means an ordered array of functions $\left\{x_{i}, X_{i L}, \theta, \psi, T_{K i}, G_{i L}, M_{K i L}, \eta, Q_{K}, f_{i}, L_{i L}, r\right\}$ which satisfy the equations of motion (2.15), the energy equation (2.19), and the constitutive relations (2.21).

Definition 3.2. A thermodynamic process will be called admissible if it also satisfies the Clausius-Duhem inequality (2.17). From the previous section it follows that the ClausiusDuhem inequality (2.17) holds for all admissible processes in $B \times I$ if and only if (2.23), (2.24), and (2.25) are satisfied.

For admissible thermodynamic processes, one may write the energy equation in reduced form

$$
\rho_{0} \theta \dot{\eta}=Q_{K, K}+\rho_{0} r
$$

Definition 3.3. One will say that $U=\left\{x_{i}, \chi_{i L}, \theta\right\}$ is an admissible state corresponding to the load $\left(f_{i}, L_{i L}, r\right)$ if $\left\{x_{i}, x_{i L}, \theta, \psi, T_{K i}, G_{i L}, M_{K i L}, \eta, Q_{K}, f_{i}, L_{i L}, r\right\}$ is an admissible thermodynamic process. The admissible state $U=\left\{x_{i}, \chi_{i L}, \theta\right\}$ is smooth if $x_{i}, \chi_{i L}$, and $\theta$ are functions of class $C^{2}$.

Let $U=\left\{x_{i}, \chi_{i L}, \theta\right\}$ and $\bar{U}=\left\{\bar{x}_{i}, \bar{X}_{i L}, \bar{\theta}\right\}$ be two smooth admissible states on $B \times I$ corresponding to the loads $\left(f_{i}, L_{i L}, r\right)$ and $\left(\bar{f}_{i}, \bar{L}_{i L}, \bar{r}\right)$, respectively. We define the function $D(\cdot)$ on $I$ by

$$
\begin{aligned}
D=\int_{B} & {\left[\frac{1}{2} \rho_{0}\left(v_{i}-\bar{v}_{i}\right)\left(v_{i}-\bar{v}_{i}\right)+\frac{1}{2} \rho_{0} I_{K L}\left(\dot{x}_{i K}-\dot{\bar{X}}_{i K}\right)\left(\dot{x}_{i L}-\dot{\bar{X}}_{i L}\right)+\rho_{0} \psi-\rho_{0} \bar{\psi}\right.} \\
& \left.-\bar{T}_{K i}\left(x_{i, K}-\bar{x}_{i, K}\right)-\bar{G}_{i K}\left(x_{i K}-\bar{x}_{i K}\right)-\bar{M}_{K i L}\left(x_{i L, K}-\bar{x}_{i L, K}\right)+\rho_{0} \eta(\theta-\bar{\theta})\right] d V,
\end{aligned}
$$

where

$$
\begin{array}{cc}
\bar{v}_{i}=\dot{\bar{x}}_{i}, \quad \bar{\psi}=\widehat{\psi}\left(\bar{x}_{i, K}, \bar{X}_{i K}, \bar{X}_{i K, M}, \bar{\theta} ; X_{K}\right), \quad \bar{Q}_{L}=\widehat{Q}_{L}\left(\bar{x}_{i, K}, \bar{X}_{i K}, \bar{X}_{i K, M}, \bar{\theta}, \bar{\theta}, K ; X_{K}\right), \\
\bar{T}_{L j}=\rho_{0} \frac{\partial \bar{\psi}}{\partial \bar{x}_{j, L}}, \quad \bar{G}_{j L}=\rho_{0} \frac{\partial \bar{\psi}}{\partial \bar{X}_{j L}}, \quad \bar{M}_{K j L}=\rho_{0} \frac{\partial \bar{\psi}}{\partial \bar{X}_{j L, K}}, \quad \bar{\eta}=-\frac{\partial \bar{\psi}}{\partial \bar{\theta}} .
\end{array}
$$


On account of (2.23) and (3.3) it is easy to see that $D$ is of quadratic order in

$$
\|\mathbf{v}-\overline{\mathbf{v}}, \dot{x}-\dot{\bar{x}}, \mathbf{F}-\overline{\mathbf{F}}, x-\bar{x}, r-\bar{\gamma}, \theta-\bar{\theta}\|_{L^{2}(B)}{ }^{\prime}
$$

where $\mathbf{F}=\left(x_{i, K}\right), \boldsymbol{X}=\left(\chi_{i K}\right)$, and $\boldsymbol{r}=\left(\chi_{i L, K}\right)$. The evolution in time of this function is described by the following.

Theorem 3.4. If $U=\left\{x_{i}, \chi_{i L}, \theta\right\}$ and $\bar{U}=\left\{\bar{x}_{i}, \bar{X}_{i L}, \bar{\theta}\right\}$ are two smooth admissible states on $B \times I$ corresponding to the loads $\left(f_{i}, L_{i L}, r\right)$ and $\left(\bar{f}_{i}, \bar{L}_{i L}, \bar{r}\right)$ in $L^{\infty}(B \times I)$, then

$$
\begin{aligned}
\dot{D}=\int_{\partial B} \Gamma d A+\int_{B}[ & \Lambda+Z+\rho_{0}\left(f_{i}-\bar{f}_{i}\right)\left(v_{i}-\bar{v}_{i}\right)+\rho_{0}\left(L_{i L}-\bar{L}_{i L}\right)\left(\dot{X}_{i L}-\dot{\bar{X}}_{i L}\right) \\
+ & \left.\frac{\rho_{0}}{\theta}(r-\bar{r})(\theta-\bar{\theta})-\frac{1}{\theta \bar{\theta}}\left(\bar{Q}_{K, K}+\rho_{0} \bar{r}\right)(\theta-\bar{\theta})^{2}\right] d V,
\end{aligned}
$$

where

$$
\begin{aligned}
& \Gamma=\left[\left(T_{K i}-\bar{T}_{K i}\right)\left(v_{i}-\bar{v}_{i}\right)+\left(M_{K i L}-\bar{M}_{K i L}\right)\left(\dot{\chi}_{i L}-\dot{\bar{\chi}}_{i L}\right)+\frac{1}{\theta}\left(Q_{K}-\bar{Q}_{K}\right)(\theta-\bar{\theta})\right] N_{K}, \\
& \Lambda=\dot{\bar{x}}_{i, K}\left[T_{K i}-\bar{T}_{K i}-\frac{\partial \bar{T}_{K i}}{\partial \bar{x}_{j, L}}\left(x_{j, L}-\bar{x}_{j, L}\right)-\frac{\partial \bar{T}_{K i}}{\partial \bar{X}_{j L}}\left(x_{j L}-\bar{x}_{j L}\right)\right. \\
& \left.-\frac{\partial \bar{T}_{K i}}{\partial \bar{X}_{j L, M}}\left(X_{j L, M}-\bar{X}_{j L, M}\right)-\frac{\partial \bar{T}_{K i}}{\partial \bar{\theta}}(\theta-\bar{\theta})\right] \\
& +\dot{\bar{X}}_{i K}\left[G_{i K}-\bar{G}_{i K}-\frac{\partial \bar{G}_{i K}}{\partial \bar{x}_{j, L}}\left(x_{j, L}-\bar{x}_{j, L}\right)-\frac{\partial \bar{G}_{i K}}{\partial \bar{x}_{j L}}\left(x_{j L}-\bar{x}_{j L}\right)-\frac{\partial \bar{G}_{i K}}{\partial \bar{X}_{j L, M}}\left(x_{j L, M}-\bar{x}_{j L, M}\right)\right. \\
& \left.-\frac{\partial \bar{G}_{i K}}{\partial \bar{\theta}}(\theta-\bar{\theta})\right] \\
& +\dot{\bar{X}}_{i L, K}\left[M_{K i L}-\bar{M}_{K i L}-\frac{\partial \bar{M}_{K i L}}{\partial \bar{x}_{j, M}}\left(x_{j, M}-\bar{x}_{j, M}\right)\right. \\
& \left.-\frac{\partial \bar{M}_{K i L}}{\partial \bar{x}_{j M}}\left(x_{j M}-\bar{x}_{j M}\right)-\frac{\partial \bar{M}_{K i L}}{\partial \bar{\chi}_{j M, N}}\left(x_{j M, N}-\bar{x}_{j M, N}\right)-\frac{\partial \bar{M}_{K i L}}{\partial \bar{\theta}}(\theta-\bar{\theta})\right] \\
& -\rho_{0} \dot{\bar{\theta}}\left[\eta-\bar{\eta}-\frac{\partial \bar{\eta}}{\partial \bar{x}_{i, K}}\left(x_{i, K}-\bar{x}_{i, K}\right)-\frac{\partial \bar{\eta}}{\partial \bar{x}_{i K}}\left(x_{i K}-\bar{x}_{i K}\right)\right. \\
& \left.-\frac{\partial \bar{\eta}}{\partial \bar{X}_{i L, K}}\left(x_{i L, K}-\bar{x}_{i L, K}\right)-\frac{\partial \bar{\eta}}{\partial \bar{\theta}}(\theta-\bar{\theta})\right] \text {, } \\
& Z=-\left(Q_{K}-\bar{Q}_{K}\right)\left(\frac{\theta-\bar{\theta}}{\theta}\right)_{, K} .
\end{aligned}
$$


Proof. From (3.2) we obtain

$$
\begin{aligned}
\dot{D}=\int_{B}\{ & \frac{d}{d t}\left[\rho_{0}\left(\psi+\theta \eta+\frac{1}{2} v_{i} v_{i}+\frac{1}{2} I_{K L} \dot{X}_{i K} \dot{X}_{i L}\right)\right] \\
& -\frac{d}{d t}\left[\rho_{0}\left(\bar{\psi}+\bar{\theta} \bar{\eta}+\frac{1}{2} \bar{v}_{i} \bar{v}_{i}+\frac{1}{2} I_{K L} \dot{\bar{X}}_{i K} \dot{\bar{X}}_{i L}\right)\right] \\
& -\rho_{0}\left(v_{i} \dot{\bar{v}}_{i}+\dot{v}_{i} \bar{v}_{i}-2 \bar{v}_{i} \dot{\bar{v}}_{i}\right)-\rho_{0} I_{K L}\left(\dot{x}_{i K} \ddot{\bar{X}}_{i L}+\ddot{X}_{i K} \dot{\bar{X}}_{i L}-2 \dot{\bar{X}}_{i K} \ddot{\bar{X}}_{i L}\right) \\
& -\dot{\bar{T}}_{K i}\left(x_{i, K}-\bar{x}_{i, K}\right)-\dot{\bar{G}}_{i K}\left(x_{i K}-\bar{x}_{i K}\right)-\dot{\bar{M}}_{K i L}\left(x_{i L, K}-\bar{X}_{i L, K}\right) \\
& -\bar{T}_{K i}\left(\dot{x}_{i, K}-\dot{\bar{x}}_{i, K}\right)-\bar{G}_{i K}\left(\dot{x}_{i K}-\dot{\bar{X}}_{i K}\right)-\bar{M}_{K i L}\left(\dot{x}_{i L, K}-\dot{\bar{X}}_{i L, K}\right) \\
& \left.-\rho_{0} \dot{\bar{\theta}}(\eta-\bar{\eta})-\rho_{0} \bar{\theta}(\dot{\eta}-\dot{\bar{\eta}})\right\} d V .
\end{aligned}
$$

Using the balance laws (2.15) and (2.19), we may write (3.9) in the form

$$
\begin{aligned}
\dot{D}=\int_{B}\{ & {\left[\left(T_{K i}-\bar{T}_{K i}\right)\left(v_{i}-\bar{v}_{i}\right)+\left(M_{K i L}-\bar{M}_{K i L}\right)\left(\dot{X}_{i L}-\dot{\bar{X}}_{i L}\right)\right]_{, k} } \\
& \left.+\rho_{0}\left(f_{i}-\bar{f}_{i}\right)\left(v_{i}-\bar{v}_{i}\right)+\rho_{0}\left(L_{i L}-\bar{L}_{i L}\right)\left(\dot{x}_{i L}-\dot{\bar{X}}_{i L}\right)+R+P\right\} d V,
\end{aligned}
$$

where

$$
\begin{aligned}
R= & -\dot{\bar{T}}_{K i}\left(x_{i, K}-\bar{x}_{i, K}\right)+\dot{\bar{x}}_{i, K}\left(T_{K i}-\bar{T}_{K i}\right)-\dot{\bar{M}}_{K i L}\left(x_{i L, K}-\bar{X}_{i L, K}\right) \\
& +\dot{\bar{X}}_{i L, K}\left(M_{K i L}-\bar{M}_{K i L}\right)-\dot{\bar{G}}_{i K}\left(x_{i K}-\bar{x}_{i K}\right)+\dot{\bar{X}}_{i K}\left(G_{i K}-\bar{G}_{i K}\right) \\
& -\rho_{0} \dot{\bar{\theta}}(\eta-\bar{\eta})+\rho_{0} \dot{\bar{\eta}}(\theta-\bar{\theta}), \\
P= & \rho_{0} r+Q_{K, K}-\rho_{0} \bar{r}-\bar{Q}_{K, K}-\rho_{0}(\dot{\bar{\eta}} \theta+\dot{\eta} \bar{\theta}-2 \dot{\bar{\eta}} \bar{\theta}) .
\end{aligned}
$$

It follows from (3.3) that

$$
\begin{gathered}
\dot{\bar{T}}_{K i}=\frac{\partial \bar{T}_{K i}}{\partial \bar{x}_{j, L}} \dot{\bar{x}}_{j, L}+\frac{\partial \bar{T}_{K i}}{\partial \bar{x}_{j L}} \dot{\bar{x}}_{j L}+\frac{\partial \bar{T}_{K i}}{\partial \bar{x}_{j L, M}} \dot{\bar{x}}_{j L, M}+\frac{\partial \bar{T}_{K i} \dot{\bar{\theta}}}{\partial \bar{\theta}}, \\
\dot{\bar{G}}_{i K}=\frac{\partial \bar{G}_{i K}}{\partial \bar{x}_{j, L}} \dot{\bar{x}}_{j, L}+\frac{\partial \bar{G}_{i K}}{\partial \bar{x}_{j L}} \dot{\bar{x}}_{j L}+\frac{\partial \bar{G}_{i K}}{\partial \bar{x}_{j L, M}} \dot{\bar{x}}_{j L, M}+\frac{\partial \bar{G}_{i K}}{\partial \bar{\theta}} \dot{\bar{\theta}}, \\
\dot{\bar{M}}_{K i L}=\frac{\partial \bar{M}_{K i L}}{\partial \bar{x}_{j, M}} \dot{\bar{x}}_{j, M}+\frac{\partial \bar{M}_{K i L}}{\partial \bar{x}_{j M}} \dot{\bar{x}}_{j M}+\frac{\partial \bar{M}_{K i L}}{\partial \bar{x}_{j M, N}} \dot{\bar{x}}_{j M, N}+\frac{\partial \bar{M}_{K i L}}{\partial \bar{\theta}}, \\
\dot{\bar{\theta}}=\frac{\partial \bar{\eta}}{\partial \bar{x}_{i, K}} \dot{\bar{x}}_{i, K}+\frac{\partial \bar{\eta}}{\partial \bar{x}_{i K}} \dot{\bar{x}}_{i K}+\frac{\partial \bar{\eta}}{\partial \bar{x}_{i K, L}} \dot{\bar{x}}_{i K, L}+\frac{\partial \bar{\eta}}{\partial \bar{\theta}} \dot{\bar{\theta}} .
\end{gathered}
$$


With the help of (3.12) and (3.3), we find that

$$
R=\Lambda
$$

On the other hand, using (3.1), we have

$$
\begin{aligned}
P= & \rho_{0} r+Q_{K, K}-\rho_{0} \bar{r}-\bar{Q}_{K, K}+\frac{\bar{\theta}}{\theta}\left(\bar{Q}_{K, K}+\rho_{0} \bar{r}\right)-\frac{\bar{\theta}}{\theta}\left(Q_{K, K}+\rho_{0} r\right) \\
& -\left(\bar{Q}_{K, K}+\rho_{0} \bar{r}\right)\left(\frac{\bar{\theta}}{\theta}-2+\frac{\theta}{\bar{\theta}}\right)=\frac{\rho_{0}}{\theta}(r-\bar{r})(\theta-\bar{\theta}) \\
& +\left[\left(Q_{K}-\bar{Q}_{K}\right) \frac{\theta-\bar{\theta}}{\theta}\right]_{, K}-\left(Q_{K}-\bar{Q}_{K}\right)\left(\frac{\theta-\bar{\theta}}{\theta}\right)_{, K}-\frac{1}{\theta \bar{\theta}}\left(\bar{Q}_{K, K}+\rho_{0} \bar{r}\right)(\theta-\bar{\theta})^{2} .
\end{aligned}
$$

Collecting (3.10), (3.13), and (3.14) and using (3.6), (3.7), and (3.8) and the divergence theorem we conclude that (3.5) holds. The proof is complete.

From (2.21), it follows that

$$
Q_{K}=\bar{Q}_{K}+\bar{K}_{K L}\left(\theta_{, L}-\bar{\theta}_{, L}\right)+\mathfrak{Q}_{K}+Q_{K^{\prime}}^{0}
$$

where

$$
\bar{K}_{K L}=\frac{\partial \widehat{Q}_{K}}{\partial \theta_{, L}}(\overline{\mathfrak{L}}), \quad \overline{\mathfrak{L}}=\left(\bar{x}_{i, M}, \bar{x}_{i M}, \bar{x}_{i M, N}, \bar{\theta}, \bar{\theta}_{, M} ; X_{M}\right),
$$

and $\mathfrak{Q}_{K}$ is given by

$$
\begin{gathered}
\mathfrak{Q}_{K}=\overline{\mathcal{B}}_{K i L}\left(x_{i, L}-\bar{x}_{i, L}\right)+\overline{\mathcal{C}}_{K i L}\left(x_{i L}-\bar{X}_{i L}\right)+\overline{\mathcal{\varepsilon}}_{K i L M}\left(x_{i L, M}-\bar{X}_{i L, M}\right)+\bar{\alpha}_{K}(\theta-\bar{\theta}), \\
\bar{乃}_{K i L}=\frac{\partial \widehat{Q}_{K}}{\partial x_{i, L}}(\overline{\mathfrak{L}}), \quad \overline{\mathcal{C}}_{K i L}=\frac{\partial \widehat{Q}_{K}}{\partial \chi_{i L}}(\overline{\mathfrak{L}}), \quad \bar{\varepsilon}_{K i L M}=\frac{\partial \widehat{Q}_{K}}{\partial \chi_{i L, M}}(\overline{\mathfrak{L}}), \quad \bar{\alpha}_{K}=\frac{\partial \widehat{Q}_{K}}{\partial \theta}(\overline{\mathfrak{L}}) .
\end{gathered}
$$

In (3.15), $Q_{K}^{0}$ is a function of order $o(\rho), \rho$ being defined by

$$
\varrho=|\mathbf{F}-\overline{\mathbf{F}}|+|\mathcal{X}-\bar{x}|+|\gamma-\bar{\gamma}|+|\theta-\bar{\theta}|+|\mathbf{g}-\overline{\mathbf{g}}|,
$$

where $\mathbf{F}=\left(x_{i, K}\right), \boldsymbol{X}=\left(\chi_{i K}\right), \boldsymbol{\gamma}=\left(\chi_{i L, K}\right)$, and $\mathbf{g}=\left(\theta_{, K}\right)$.

Let us introduce the following definition for a definite heat conductor material (see Chiriţă [19]). 
Definition 3.5. One says that the admissible state $\bar{U}$ resides in the region where the material is a definite heat conductor if

$$
\bar{k}_{K L} \zeta_{K} \zeta_{L}>0
$$

for any nonzero $\zeta_{K}$, where $\bar{k}_{K L}$ is given by

$$
\bar{k}_{K L}=\frac{1}{2}\left(\bar{K}_{K L}+\bar{K}_{L K}\right) .
$$

We introduce the following notation:

$$
y(t)=\|(\mathbf{F}-\overline{\mathbf{F}}, x-\bar{x}, \boldsymbol{r}-\bar{\gamma}, \theta-\bar{\theta})(\cdot, t)\|_{L^{2}(B),} \quad t \in I .
$$

Theorem 3.6. Let $\bar{U}=\left\{\bar{x}_{i}, \bar{X}_{i L}, \bar{\theta}\right\}$ be a smooth admissible state residing in the region where the material is a definite conductor of heat. Then there exist the positive constants $\delta, m_{1}$, and $m_{2}$ with the following property: if $U=\left\{x_{i}, \chi_{i L}, \theta\right\}$ is any smooth admissible process defined on $B \times I$, such that

$$
\varrho=|\mathbf{F}-\overline{\mathbf{F}}|+|x-\bar{x}|+|\gamma-\bar{\gamma}|+|\theta-\bar{\theta}|+|\mathbf{g}-\overline{\mathbf{g}}|<\delta,
$$

on $B \times I$, then

$$
\int_{B} Z d V \leq-m_{1}\|(\mathbf{g}-\overline{\mathbf{g}})(\cdot, t)\|_{L^{2}(B)}^{2}+m_{2} y^{2}(t), \quad t \in I
$$

Proof. In view of (3.8), (3.15), and (3.21), it follows that

$$
\begin{aligned}
Z & =-\frac{1}{\theta}\left(Q_{K}-\bar{Q}_{K}\right)\left(\theta_{, K}-\bar{\theta}_{, K}\right)+\frac{1}{\theta^{2}} \theta_{, K}\left(Q_{K}-\bar{Q}_{K}\right)(\theta-\bar{\theta}) \\
& =-\frac{1}{\theta} \bar{k}_{K L}\left(\theta_{, K}-\bar{\theta}_{, K}\right)\left(\theta_{, L}-\bar{\theta}_{, L}\right)+\mathfrak{K}+o\left(\rho^{2}\right),
\end{aligned}
$$

where

$$
\mathfrak{K}=-\frac{1}{\theta} \mathfrak{Q}_{K}\left(\theta_{, K}-\bar{\theta}_{, K}\right)+\frac{1}{\theta^{2}} \theta_{, K}\left[\bar{K}_{K L}\left(\theta_{, L}-\bar{\theta}_{, L}\right)+\mathfrak{Q}_{K}\right](\theta-\bar{\theta}) .
$$

Using the arithmetic-geometric inequality

$$
2 a b \leq a^{2} \beta^{2}+b^{2} \beta^{-2}, \quad \text { for all nonzero constant } \beta,
$$


Schwarz's inequality, and (3.17), we deduce

$$
\begin{aligned}
2 \int_{B} \mathfrak{K} d V \leq & \left(\beta_{1}^{2}+\beta_{2}^{2}+\beta_{3}^{2}+\beta_{4}^{2}\right)\|(\mathbf{g}-\overline{\mathbf{g}})(\cdot, t)\|_{L^{2}(B)}^{2} \\
& +\left(M_{1}^{2} \beta_{1}^{-2}+\gamma_{1}^{2}\right)\|(\mathbf{F}-\overline{\mathbf{F}})(\cdot, t)\|_{L^{2}(B)}^{2}+\left(M_{2}^{2} \beta_{2}^{-2}+\gamma_{2}^{2}\right)\|(x-\bar{x})(\cdot, t)\|_{L^{2}(B)}^{2} \\
& +\left(M_{3}^{2} \beta_{3}^{-2}+\gamma_{3}^{2}\right)\|(\gamma-\bar{\gamma})(\cdot, t)\|_{L^{2}(B)}^{2} \\
& +\left(M_{4}^{2} \beta_{4}^{-2}+N_{1}^{2} \gamma_{1}^{-2}+N_{2}^{2} \gamma_{2}^{-2}+N_{3}^{2} \gamma_{3}^{-2}+N^{2}\right)\|(\theta-\bar{\theta})(\cdot, t)\|_{L^{2}(B)^{\prime}}^{2}
\end{aligned}
$$

where $\beta_{p},(p=1,2,3,4)$ and $\gamma_{s},(s=1,2,3)$ are arbitrary nonzero constants and

$$
\begin{gathered}
M_{1}=\max \left|\theta^{-1} \overline{\bar{B}}\right|, \quad M_{2}=\max \left|\theta^{-1} \overline{\mathcal{C}}\right|, \quad M_{3}=\max \left|\theta^{-1} \overline{\mathcal{\varepsilon}}\right|, \\
M_{4}=\max \left|\theta^{-1} \bar{\alpha}+\theta^{-2} \bar{K}^{T} \mathbf{g}\right|, \quad N_{1}=\max \left|\theta^{-2} \overline{\mathcal{B}}^{T} \mathbf{g}\right|, \quad N_{2}=\max \left|\theta^{-2} \overline{\mathcal{C}}^{T} \mathbf{g}\right|, \\
N_{3}=\max \left|\theta^{-2} \overline{\mathcal{\varepsilon}}^{T} \mathbf{g}\right|, \quad N^{2}=2 \max \left|\theta^{-2} \bar{\alpha} \mathbf{g}\right| .
\end{gathered}
$$

In view of (3.20), we conclude that there exists a positive constant $\lambda$ such that

$$
-\int_{B} \frac{1}{\theta} \bar{k}_{K L}\left(\theta_{, K}-\bar{\theta}_{, K}\right)\left(\theta_{, L}-\bar{\theta}_{, L}\right) d V \leq-\lambda\|(\mathbf{g}-\overline{\mathbf{g}})(\cdot, t)\|_{L^{2}(B)}^{2}, \quad t \in I .
$$

Collecting (3.25), (3.28), and (3.30), we conclude that there exists a positive constant $\delta$ with the property that whenever (3.23) holds, we have

$$
\begin{aligned}
\int_{B} Z d V \leq & -m_{1}\|(\mathbf{g}-\overline{\mathbf{g}})(\cdot, t)\|_{L^{2}(B)}^{2}+c_{1}\|(\mathbf{F}-\overline{\mathbf{F}})(\cdot, t)\|_{L^{2}(B)}^{2} \\
& +c_{2}\|(\boldsymbol{X}-\bar{x})(\cdot, t)\|_{L^{2}(B)}^{2}+c_{3}\|(\boldsymbol{r}-\bar{r})(\cdot, t)\|_{L^{2}(B)}^{2}+c_{4}\|(\theta-\bar{\theta})(\cdot, t)\|_{L^{2}(B)}^{2}
\end{aligned}
$$

where

$$
\begin{gathered}
m_{1}=\lambda-\frac{1}{2}\left(\beta_{1}^{2}+\beta_{2}^{2}+\beta_{3}^{2}+\beta_{4}^{2}\right), \quad 2 c_{1}=M_{1}^{2} \beta_{1}^{-2}+\gamma_{1}^{2}, \quad 2 c_{2}=M_{2}^{2} \beta_{2}^{-2}+\gamma_{2}^{2}, \\
2 c_{3}=M_{3}^{2} \beta_{3}^{-2}+\gamma_{3}^{2}, \quad 2 c_{4}=M_{4}^{2} \beta_{4}^{-2}+N_{1}^{2} \gamma_{1}^{-2}+N_{2}^{2} \gamma_{2}^{-2}+N_{3}^{2} \gamma_{3}^{-2}+N^{2} .
\end{gathered}
$$

Now, taking the constants $\beta_{p},(p=1,2,3,4)$ such that $m_{1}>0$ and setting

$$
m_{2}=\max \left\{c_{1}, c_{2}, c_{3}, c_{4}\right\},
$$

from (3.22) and (3.31), we obtain (3.24), and the proof is complete. 
Following [18], we introduce the following.

Definition 3.7. One says that the admissible state $\bar{U}=\left\{\bar{x}_{i}, \bar{X}_{i L}, \bar{\theta}\right\}$ resides in the convexity region of the internal energy if the following two conditions are satisfied:

(i) for each $(\mathbf{X}, t) \in \bar{B} \times I$, there exists a positive constant $\mu$ such that

$$
\begin{aligned}
& \frac{\partial \bar{\psi}}{\partial \bar{x}_{i, K} \bar{x}_{j, L}} \Upsilon_{i K} \Upsilon_{j L}+\frac{\partial \bar{\psi}}{\partial \bar{X}_{i K} \bar{X}_{j L}} \Omega_{i K} \Omega_{j L}+\frac{\partial \bar{\psi}}{\partial \bar{\chi}_{i K, M} \bar{X}_{j L, N}} \Phi_{i K M} \Phi_{j L N}+2 \frac{\partial \bar{\psi}}{\partial \bar{x}_{i, K} \bar{X}_{j L}} \Upsilon_{i K} \Omega_{j L} \\
& \quad+2 \frac{\partial \bar{\psi}}{\partial \bar{x}_{i, K} \bar{X}_{j L, M}} \Upsilon_{i K} \Phi_{j L M}+2 \frac{\partial \bar{\psi}}{\partial \bar{X}_{i K} \bar{X}_{j L, M}} \Omega_{i K} \Phi_{j L M} \geq \mu\left(\Upsilon_{i K} \Upsilon_{i K}+\Omega_{i K} \Omega_{i K}+\Phi_{i K L} \Phi_{i K L}\right),
\end{aligned}
$$

for all $\Upsilon_{i K}, \Omega_{i K}$, and $\Phi_{i K L}$

(ii) and

$$
\frac{\partial \bar{\eta}}{\partial \bar{\theta}}>0
$$

Our study on stability and uniqueness is based on the following Gronwall-type inequality [18].

Lemma 3.8. Assume that the nonnegative functions $z(t) \in L^{\infty}[0, s]$ and $g(t) \in L^{1}[0, s]$ satisfy the inequality

$$
z^{2}(\tau) \leq M^{2} z^{2}(0)+2 \int_{0}^{\tau}\left[(\alpha+2 \beta \tau) z^{2}(t)+N g(t) z(t)\right] d t, \quad \tau \in[0, s]
$$

with $\alpha, \beta, M$, and $N$ being nonnegative constants. Then

$$
z(s) \leq\left[M z(0)+N \int_{0}^{s} g(t) d t\right] \exp \left(\sigma s+\beta s^{2}\right)
$$

where $\sigma=\alpha+\beta / \alpha$.

Now, we are ready to state the following stability result.

Theorem 3.9. Let $\bar{U}=\left\{\bar{x}_{i}, \bar{X}_{i K}, \bar{\theta}\right\}$ be a smooth admissible state on $B \times I$ corresponding to the loading $\left(\bar{f}_{i}, \bar{L}_{i L}, \bar{r}\right) \in L^{\infty}(B \times I)$ and residing in the region where the internal energy is a convex function and the material is a definite conductor of heat. Then there exist the positive constants $\widetilde{\delta}_{0}, \alpha_{0}, M_{0}$, and $N_{0}$ with the following property: if $U=\left\{x_{i}, x_{i K}, \theta\right\}$ is any smooth admissible state on $B \times I$ corresponding to the loading $\left(f_{i}, L_{i K}, r\right) \in L^{\infty}(B \times I)$, such that

$$
\varrho=|\mathbf{F}-\overline{\mathbf{F}}|+|\boldsymbol{X}-\overline{\boldsymbol{X}}|+|\boldsymbol{\gamma}-\bar{\gamma}|+|\theta-\bar{\theta}|+|\mathbf{g}-\overline{\mathbf{g}}|<\tilde{\delta}_{0}
$$


on $B \times I$, and

$$
\begin{array}{r}
{\left[\left(T_{K i}-\bar{T}_{K i}\right)\left(v_{i}-\bar{v}_{i}\right)+\left(M_{K i L}-\bar{M}_{K i L}\right)\left(\dot{X}_{i L}-\dot{\bar{X}}_{i L}\right)+\frac{1}{\theta}\left(Q_{K}-\bar{Q}_{K}\right)(\theta-\bar{\theta})\right] N_{K}=0,} \\
\text { on } \partial B \times I,
\end{array}
$$

then for any $s \in I$, one has

$$
z_{0}(s) \leq\left[M_{0} z_{0}(0)+N_{0} \int_{0}^{s} g_{0}(t) d t\right] \exp \left(\alpha_{0} s\right)
$$

where

$$
\begin{gathered}
z_{0}(s)=\|(\mathbf{v}-\overline{\mathbf{v}}, \mathbf{F}-\overline{\mathbf{F}}, \boldsymbol{X}-\bar{X}, \dot{X}-\dot{\bar{X}}, \boldsymbol{\gamma}-\bar{\gamma}, \theta-\bar{\theta})(\cdot, s)\|_{L^{2}(B)^{\prime}} \\
g_{0}(s)=\|(\mathbf{f}-\overline{\mathbf{f}}, \mathbf{L}-\overline{\mathbf{L}}, r-\bar{r})(\cdot, s)\|_{L^{2}(B)} .
\end{gathered}
$$

Proof. From (3.6) and (3.39), we have $\Gamma=0$. In view of (3.5), (3.7), (3.24), and Schwarz inequality, it follows that there exist the positive constants $\delta, v_{1}$, and $v_{2}$ such that whenever (3.23) holds, we have

$$
\dot{D}(t) \leq v_{1} y^{2}(t)+v_{2} g_{0}(t) w(t), \quad t \in I
$$

where $y(t)$ is defined in (3.22) and

$$
w(t)=\|(\mathbf{v}-\overline{\mathbf{v}}, \dot{X}-\dot{\bar{X}}, \theta-\bar{\theta})(\cdot, t)\|_{L^{2}(B)} .
$$

Let us fix $s \in I$ and integrate (3.42) over $[0, \tau]$, with $\tau \in[0, s]$. Then, we have

$$
D(\tau) \leq D(0)+v_{1} \int_{0}^{\tau} z_{0}^{2}(t) d t+v_{2} \int_{0}^{\tau} g_{0}(t) z_{0}(t) d t
$$

Here we used the inequalities $y(t) \leq z_{0}(t)$ and $w(t) \leq z_{0}(t), t \in I$. 
On the other hand, in view of (2.23) and (3.3), we obtain

$$
\begin{aligned}
\rho_{0} \psi-\rho_{0} \bar{\psi}- & \bar{T}_{K i}\left(x_{i, K}-\bar{x}_{i, K}\right)-\bar{G}_{i K}\left(x_{i K}-\bar{x}_{i K}\right)-\bar{M}_{K i L}\left(x_{i L, K}-\bar{x}_{i L, K}\right)+\rho_{0} \eta(\theta-\bar{\theta}) \\
= & \frac{\rho_{0}}{2}\left[\frac{\partial \bar{\psi}}{\partial \bar{x}_{i, K} \partial \bar{x}_{j, L}}\left(x_{i, K}-\bar{x}_{i, K}\right)\left(x_{j, L}-\bar{x}_{j, L}\right)\right. \\
& +\frac{\partial \bar{\psi}}{\partial \bar{x}_{i K} \partial \bar{x}_{j L}}\left(x_{i K}-\bar{x}_{i K}\right)\left(x_{j L}-\bar{x}_{j L}\right) \\
& +\frac{\partial \bar{\psi}}{\partial \bar{x}_{i K, M} \partial \bar{x}_{j L, N}}\left(x_{i K, M}-\bar{x}_{i K, M}\right)\left(x_{j L, N}-\bar{x}_{j L, N}\right) \\
& +2 \frac{\partial \bar{\psi}}{\partial \bar{x}_{i, K} \partial \bar{x}_{j L}}\left(x_{i, K}-\bar{x}_{i, K}\right)\left(x_{j L}-\bar{x}_{j L}\right) \\
& +2 \frac{\partial \bar{\psi}}{\partial \bar{x}_{i, K} \partial \bar{X}_{j L, M}}\left(x_{i, K}-\bar{x}_{i, K}\right)\left(x_{j L, M}-\bar{x}_{j L, M}\right) \\
& \left.+2 \frac{\partial \bar{\psi}}{\partial \bar{x}_{i K} \partial \bar{x}_{j L, M}}\left(x_{i K}-\bar{x}_{i K}\right)\left(x_{j L, M}-\bar{x}_{j L, M}\right)\right] \\
+ & \frac{1}{2} \frac{\partial \bar{\eta}}{\partial \bar{\theta}}(\theta-\bar{\theta})^{2}+o\left(\left|\mathbf{F}-\overline{\mathbf{F}}^{2}+\right| x-\left.\bar{x}\right|^{2}+|r-\bar{r}|^{2}+|\theta-\bar{\theta}|^{2}\right) .
\end{aligned}
$$

It follows from (3.2), (3.34), (3.35), and (3.45) that there exist the positive constants $\delta_{0}$ and $v_{3}$ such that whenever

$$
|\mathbf{F}-\overline{\mathbf{F}}|+|x-\bar{x}|+|\gamma-\bar{\gamma}|+|\theta-\bar{\theta}|<\delta_{0}
$$

we have

$$
v_{3} z_{0}(t) \leq 2 D(t), \quad t \in I \text {. }
$$

Setting $\widetilde{\delta}_{0}=\min \left(\delta, \delta_{0}\right)$ in (3.38), from (3.44) and (3.47), we obtain

$$
v_{3} z_{0}^{2}(\tau) \leq 2 D(0)+2 v_{1} \int_{0}^{\tau} z_{0}^{2}(t) d t+2 v_{2} \int_{0}^{\tau} g_{0}(t) z_{0}(t) d t
$$

Using the estimate

$$
D(0) \leq v_{4} z_{0}(0), \quad v_{4}>0
$$

and the notations

$$
M_{0}^{2}=\frac{2 v_{4}}{v_{3}}, \quad \alpha_{0}=\frac{v_{1}}{v_{3}}, \quad N_{0}=\frac{v_{2}}{v_{3}},
$$


then (3.48) implies that

$$
z_{0}^{2}(\tau) \leq M_{0}^{2} z_{0}(0)+2 \int_{0}^{\tau}\left[\alpha_{0} z_{0}^{2}(t) d t+N_{0} g_{0}(t) z_{0}(t)\right] d t
$$

An application of Lemma 3.8 completes the proof.

A direct consequence of the above theorem is the following uniqueness result.

Theorem 3.10. Let $U$ and $\bar{U}$ be as in Theorem 3.9. Assume that the corresponding body loads coincide on $B \times I$ and $U$ and $\bar{U}$ originate from the same state, namely,

$$
\begin{array}{ccc}
x_{i}(\mathbf{X}, 0)=\bar{x}_{i}(\mathbf{X}, 0), \quad v_{i}(\mathbf{X}, 0)=\bar{v}_{i}(\mathbf{X}, 0), \quad X_{i L}(\mathbf{X}, 0)=\bar{X}_{i L}(\mathbf{X}, 0), \quad \mathbf{X} \in B . \\
\dot{X}_{i L}(\mathbf{X}, 0)=\dot{\bar{X}}_{i L}(\mathbf{X}, 0), \quad \theta(\mathbf{X}, 0)=\bar{\theta}(\mathbf{X}, 0), &
\end{array}
$$

Then, $U$ and $\bar{U}$ coincide on $B \times I$.

\section{Acknowledgment}

This work was supported by CNCSIS-UEFISCSU, project PN II-RU TE code 184, no. 86/30.07.2010.

\section{References}

[1] A. C. Eringen and E. S. Şuhubi, “Nonlinear theory of simple microelastic solids I \& II," International Journal of Engineering Science, vol. 2, pp. 189-404, 1964.

[2] A. C. Eringen, Microcontinuum Field Theories. I. Foundations and Solids, Springer, New York, NY, USA, 1999.

[3] E. Soós, "Uniqueness theorems for homogeneous, isotropic, simple elastic and thermoelastic materials having a microstructure," International Journal of Engineering Science, vol. 7, pp. 257-268, 1969.

[4] D. Ieşan, "On the micromorphic thermoelasticity," International Journal of Engineering Science, vol. 40, no. 5, pp. 549-567, 2002.

[5] M. Maugin, "Un principe variationnel pour des milieu micromorphic non dissipative," Comptes Rendus de l'Académie des Sciences, Paris, vol. 271, pp. 807-801, 1970.

[6] L. Nappa, "Variational principles in micromorphic thermoelasticity," Mechanics Research Communications, vol. 28, no. 4, pp. 405-412, 2001.

[7] R. Teisseyre, "Some seismic phenomena in the light of the symmetric micromorphic theory," Journal of Technical Physics, vol. 23, no. 1, pp. 95-99, 1982.

[8] L. Dresen, J. Kozák, A. Špičák, L. Waniek, R. Teisseyre, and A. Zátopek, “Wave propagation in physical models of micromorphic media," Studia Geophysica et Geodaetica, vol. 28, no. 3, pp. 272-285, 1984.

[9] R. Teisseyre, L. Dresden, J. Kozak, and L. Waniek, "Physical properties of micromorphic medium: theory and experiment," Acta Geophysica Polonica, vol. 33, no. 4, pp. 344-356, 1985.

[10] Y. Chen and J. D. Lee, "Determining material constants in micromorphic theory through phonon dispertion relations," International Journal of Engineering Science, vol. 41, no. 3-5, pp. 871-886, 2003.

[11] R. J. Twiss and A. C. Eringen, "Theory of mixtures for micromorfic materials I," International Journal of Engineering Science, vol. 9, pp. 1019-1044, 1971.

[12] R. J. Twiss and A. C. Eringen, "Theory of mixtures for micromorfic materials II. Elastic constitutive equations," International Journal of Engineering Science, vol. 10, pp. 437-465, 1972

[13] A. C. Eringen, "Balance laws of micromorphic mechanics," International Journal of Engineering Science, vol. 8, no. 10, pp. 819-828, 1970. 
[14] A. C. Eringen, "Continuum theory of micromorphic electromagnetic thermoelastic solids," International Journal of Engineering Science, vol. 41, no. 7, pp. 653-665, 2003.

[15] J. D. Lee and Y. Chen, "Constitutive relations of micromorphic thermoplasticity," International Journal of Engineering Science, vol. 41, pp. 1041-1057, 2003.

[16] J. D. Lee and Y. Chen, "Material forces in micromorphic thermoelastic solids," Philosophical Magazine, vol. 85, pp. 3897-3910, 2005.

[17] D. Ieşan and L. Nappa, "On the theory of heat for micromorphic bodies," International Journal of Engineering Science, vol. 43, no. 1-2, pp. 17-32, 2005.

[18] C. M. Dafermos, "The second law of thermodynamics and stability," Archive for Rational Mechanics and Analysis, vol. 70, no. 2, pp. 167-179, 1979.

[19] S. Chiriţă, "Uniqueness and continuous data dependence in dynamical problems of nonlinear thermoelasticity," Journal of Thermal Stresses, vol. 5, no. 3-4, pp. 331-346, 1982.

[20] D. Ieşan, "On the nonlinear theory of interacting micromorphic materials," International Journal of Engineering Science, vol. 42, no. 19-20, pp. 2135-2145, 2004.

[21] D. Ieşan, "Continuous dependence in a nonlinear theory of viscoelastic porous mixtures," International Journal of Engineering Science, vol. 44, no. 17, pp. 1127-1145, 2006.

[22] D. Ieşan and L. Nappa, "On the theory of viscoelastic mixtures and stability," Mathematics and Mechanics of Solids, vol. 13, no. 1, pp. 55-80, 2008.

[23] D. Ieşan, Thermoelastic Models of Continua, vol. 118 of Solid Mechanics and Its Applications, Kluwer Academic Publishers, Dordrecht, The Netherlands, 2004. 


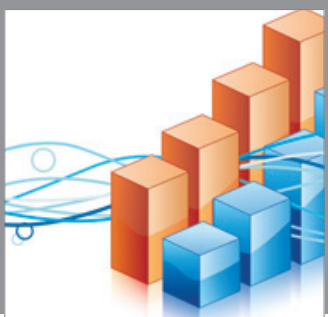

Advances in

Operations Research

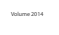

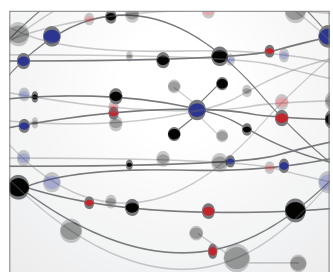

\section{The Scientific} World Journal
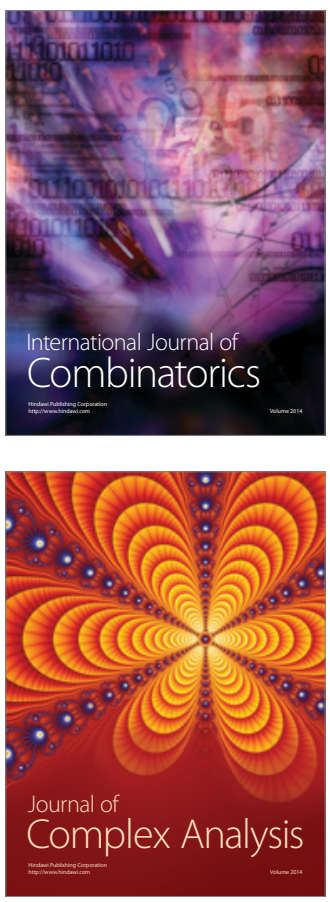

International Journal of

Mathematics and

Mathematical

Sciences
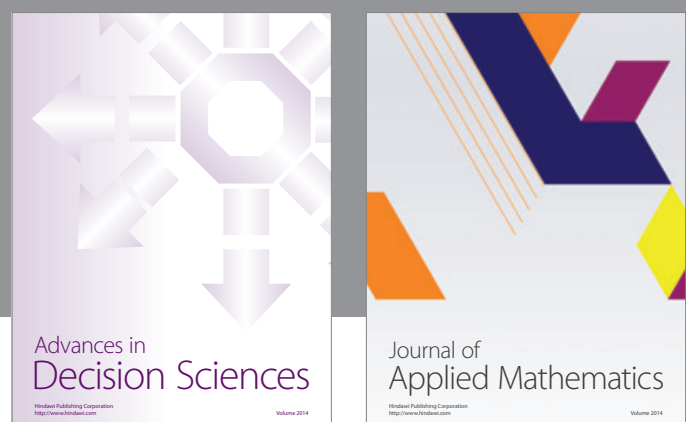

Journal of

Applied Mathematics
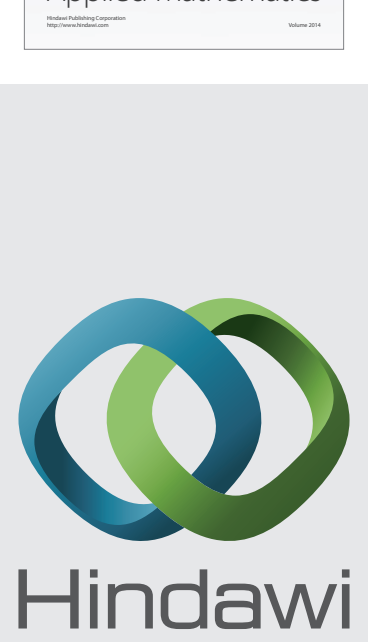

Submit your manuscripts at http://www.hindawi.com
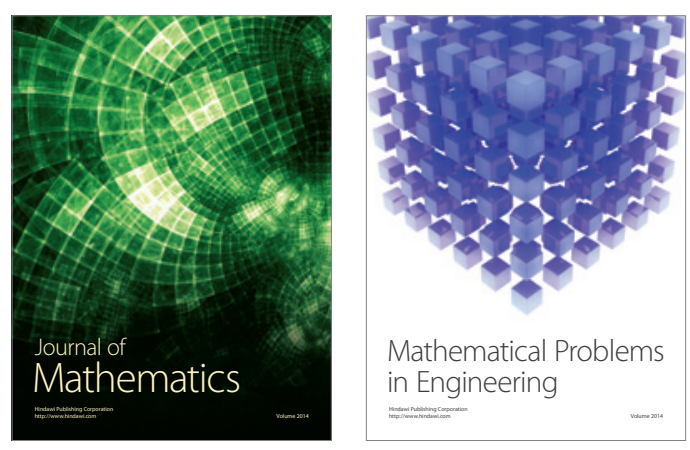

Mathematical Problems in Engineering
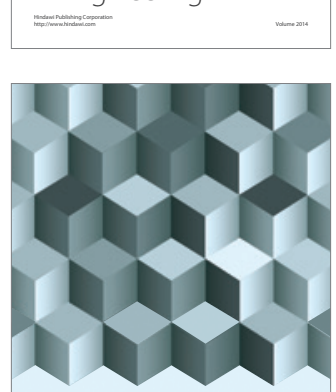

Journal of

Function Spaces
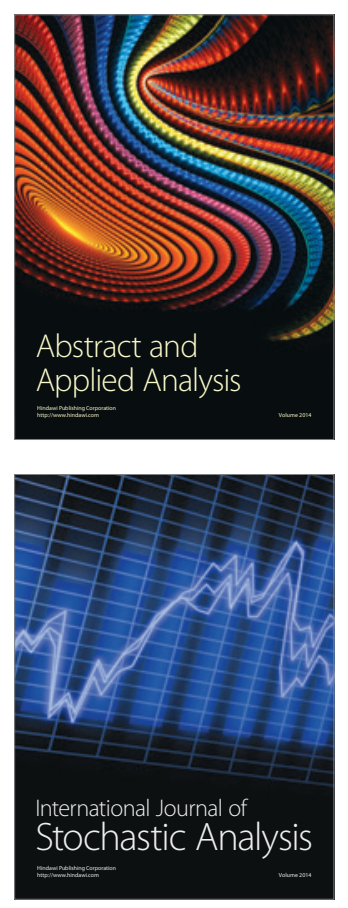

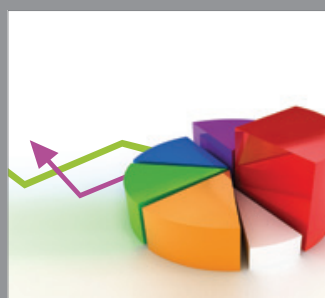

ournal of

Probability and Statistics

Promensencen
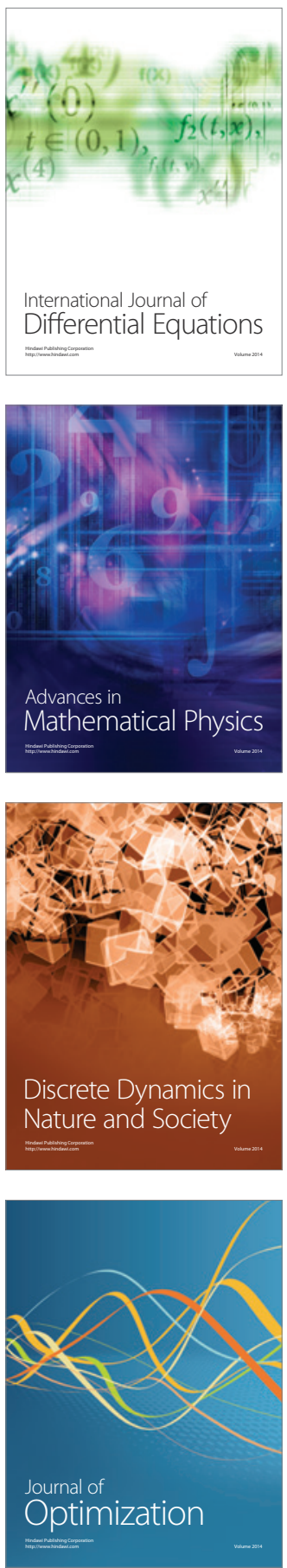